\title{
密歇根神经篮查量表在中国2型糖尿病患者周围神经病 变筱查中的应用分析
}

唐美丽 刘雪彦* 周佩如 陈庆玲 黄洁微 刘宜苑 暨南大学附属第一医院, 广东 510630

摘 要: 目的: 探讨密歇根神经篮查量表 (MNSI) 在糖尿病患者周围神经病变篎查中诊断价值。方法: 对206例 2型糖尿病患者进行密歇根神经篎查量表 (体格检查和问卷评分) 篎查, 以神经肌电图检查为诊断糖尿病周围神经病 变 (DPN) 的标准, 将患者分为DPN组和非DPN组, 采用诊断试验和ROC曲线分析的方法, 评价密歇根神经篎查量表 的诊断价值。结果: MNSI体格检查和问卷ROC曲线下面积 ( AUC) 分别为 0.828 和 $0.838(P<0.01)$, 两者的诊断截 断点为 2 分时敏感性和特异性之和最大。结论：MNSI体格检查或问卷评分, 截点为 2 分时, 与金标准肌电图诊断一致 性较好，特别适用于偏远地区或二线城市等基层地区的糖尿病患者周围神经病变的大规模查。

关键词: 2型糖尿病; 周围神经病变；密歇根神经篎查量表

\section{一、前言}

糖尿病周围神经病变 (DPN) 是指周围神经功能障碍, 包含脊神经、颅神经及植物神经病变, 其中以远端对称性 多发性神经病变 (Distal Symmetric Poly Neuropathy, DSPN) 最具代表性 ${ }^{[1]}$ 。多以肢体远端感觉异常为首发症状, 可 呈现手套. 袜套样感觉障碍, 临床可表现为双侧肢体疼痛、麻木、感觉异常、肌无力、肌萎缩、眼睑闭合、面㿈、泌 汗异常等 ${ }^{[2]}$ 。糖尿病周围神经病变是糖尿病最常见的慢性并发症之一, 其发病与长期的高血糖等因素相关, 可累及感 觉、运动或自主神经, 出现感觉异常、疼痛和睡眠障碍, 而也有部分糖尿病周围神经病变的患者没有临床症状, 导致 诊治延误, 成为足部溃疡和截肢的重要因素 ${ }^{[1]}$ 。因此, 早期诊断糖尿病周围神经病变和积极的干预, 做好糖尿病二级 和三级预防，对改善患者临床结局具有重要意义。

中国2型糖尿病防治指南推荐简易篮查糖尿病周围神经的方法依赖于临床症状 (疼痛、麻木等) 和五项检查 (压 力觉、振动觉、针刺痛觉、温度觉和踝反射), 也有学者研究证明用踝反射、振动觉、温度觉三项联合为最优组合 简易笁查方法 ${ }^{[3]}$ 。有典型症状者易于发现和诊断, 无症状者需要通过体格检查或神经电生理检查做出诊断 ${ }^{\left[{ }^{2}\right]}$ 。目前, 肌电图 ( $\mathrm{EMG}$ ) 可以避免主观偏见 ${ }^{[4,5]}$, 是周围神经病变的最佳替代诊断方法。美国糖尿病学会 (American Diabetes Association, $\mathrm{ADA}$ ) 指出: 糖尿病患者进行DSPN的篮查应至少使用以下五种筛查方法之一 (临床症状, 临床检查, 神经传导NCS、定量感觉和自主症状) ${ }^{[6]}$ 。密歇根神经笁查量表 (Michigan Neuropathy Screening Instrument, MNSI) 在国外普遍被应用于糖尿病周围神经病变的篮查, MNSI包含两部分, 问卷和体格检查 ${ }^{[6-8]}$ 。国外研究已证实MNSI体 格检查评分 $\geqslant 2$ 分对诊断DPN的准确率高, 问卷评分 $\geqslant 4$ 分被严格认为存在周围神经病变, $\geqslant 7$ 分被保守认为存在周 围神经病变 ${ }^{[8-10]}$ 。但在国内较少学者探索体格检查评分系统诊断截点的可靠性, 且MNSI问卷与DSPN关系的报道尚少 见。本研究旨在探讨MNSI问卷和体格检查在糖尿病患者DPN篮查中的应用价值。

\section{二、研究对象}

采用整群便利抽样方法选取2018年12月至2019年4月在暨南大学附属第一医院内分泌科住院的2型糖尿病患者。

(一) 纳人标准

根据1999年WHO糖尿病诊断标准确诊的2型糖尿病患者; 年龄 $\geqslant 18$ 岁; 神志清醒, 语言沟通无障碍; 自愿参与本 研究并同意接受周围神经筛查者。

(二) 排除标准

无法言语沟通者; 1型糖尿病患者; 有可能因颈椎、腰椎病变引起的神经病变患者; 中途退出者或拒绝本研究者。

*通讯作者: 刘雪彦, 1981年10月, 女, 河南周口人, 汉族, 现任暨南大学附属第一医院内分泌副护长, 主管护 师, 硕士。研究方向: 糖尿病足管理。

基金项目：2016年广东省省科技发展专项资金，糖尿病智能可穿戴设备与并发症防控云平台（项目编号： 2016B010108008)。 


\section{三、研究方法}

本研究采用了横断面调查研究方法, 研究人员以肌电图/神经传导速度 ( $\mathrm{EMG} / \mathrm{NCV})$ 为诊断DPN的标准, 同时对 患者进行MNSI筛查，来判断MNSI诊断DPN的价值。收集相关资料如下。

(一) 神经电生理检查

由医院取得肌电图操作资格的技师测定，使用NDI-200P+肌电图仪/诱发电位仪 (上海波塞冬医疗电子仪器有限公 司）, 在安静的室内环境中进行检查。皮肤温度为 $28^{\circ} \mathrm{C} \sim 30^{\circ} \mathrm{C}$, 室温为 $20^{\circ} \mathrm{C} \sim 25^{\circ} \mathrm{C}$ 。测量四肢运动神经和感觉神经的传 导速度和波幅，以判别周围神经是否存在病变。

(二) MNSI笁查

由研究人员在患者住院期间对其进行MNSI笁查，包括问卷（15项）和体格检查（4项）两部分，详见表1和表2。

\section{表1 MNSI问卷部分}

\begin{tabular}{ccc}
\hline 序号 & 项目 & 否 \\
\hline 1 & 你的下肢或足部有麻木感吗? & \\
2 & 你的下肢或足部曾经有过灼痛的感觉吗? & \\
3 & 你的双足有感觉过敏的现象吗? \\
4 & 你的下肢或双足出现过肌肉痛性痉孪的现象吗? & \\
5 & 你的下肢或双足出现过刺痛的感觉吗? \\
6 & 当被裖接触皮肤时你有被刺痛的感觉吗? \\
7 & 当你淋浴时, 能清楚的感知水温的变化吗? \\
9 & 你曾经有过足部溃疡吗? \\
10 & 你的医生诊断过你患有糖尿病神经病变吗? \\
11 & 你大部分时间会感到虚弱无力吗? \\
13 & 你的症状在夜间是否会更严重? \\
14 & 你的下肢在走路时受过伤吗? \\
15 & 你行走时能感觉到你的双足吗? \\
\hline
\end{tabular}

表2 MNSI体格检查部分

\begin{tabular}{cc}
\hline 项目 & 计分值 \\
\hline 外观 & 正常 $=0$, 异常 $($ 畸形、皮肤干燥、胼胝、感染、溃疡 $)=1$ 分 \\
踝反射 & 存在 $=0$, 重扣击出现 $=0.5$ 分, 消失 $=1$ 分 \\
足部溃疡 & 无 $=0$ 分, 有 $=1$ 分 \\
振动觉 & 存在 $=0$ 分, 减弱 $=0.5$ 分, 消失 $=1$ 分 \\
\hline
\end{tabular}

问卷由研究人员提问，患者进行回答，保证问卷完整性和有效性。研究人员对患者亦进行足部外观、踝反射、足 部是否存在溃疡和振动觉四项体格检查。问卷评分满分为 13 分, 体格检查满分为 8 分 ${ }^{[1]}$ 。问题 4 和问题 10 不计人问卷总 分。问题 7 和问题 13 当患者做出肯定回答时为 0 分, 做出否定回答时为 1 分, 其余的 11 项问题当患者做出肯定回答时为 1 分，做出否定回答时为 0 分。体检评分满分8分（包括左右两侧）。

(三) 统计学方法

采用SPSS19.0软件进行统计学分析, 计量数据以 $\chi^{2} \pm s$ 表示; 计数资料以例数（百分比） $[n(\%)]$ 表示, 采用 $\chi^{2}$ 检验; 以肌电图诊断DPN为金标准，同时测试患者MNSI问卷和体格检查不同分值水平下DPN和非DPN的数量, 做 出受试者工作特征曲线（ROC），计算问卷和体格检查不同分值查对DPN预测的敏感性、特异性、ROC曲线下面积 ( AUC ) 及 $95 \% \mathrm{CI}$ ，找出最优的截点。并以最优截点为界，用四格表分别计算MNSI问卷和体格检查计算与金标准肌 电图诊断DPN的kaPPa值，判断其相关性。通过AUC和kaPPa值来判断分析MNSI诊断DPN的价值。

\section{四、结果}

共纳人糖尿病患者 206 例, 其中男 97 例、女 109 例, 平均年龄为 $59.79 \pm 14.25$ 岁, 平均病程为 $8.82 \pm 7.46$ 年。肌电图结果 显示异常的可判定为DPN有90例，正常结果非DPN者有106例。MNSI体格检查不同分值时DPN和非DPN的例数见表3。 
表3 MNSI体格检查的得分情况

\begin{tabular}{ccc}
\hline 得分 & DPN例数 (百分比) & 非DPN例数(百分比) \\
\hline$\geqslant 0$ & $15(16.7 \%)$ & $98(84.5 \%)$ \\
$\geqslant 2$ & $66(73.3 \%)$ & $13(11.2 \%)$ \\
$\geqslant 4$ & $7(7.8 \%)$ & $4(3.4 \%)$ \\
$\geqslant 6$ & $2(2.2 \%)$ & $1(0.9 \%)$ \\
$\geqslant 8$ & $0(0 \%)$ & $0(0 \%)$ \\
\hline
\end{tabular}

MNSI体格检查选取1 (相当于原始数据2分) 为截断点时, 此时的灵敏度 ( $83.3 \%$ ) 与特异度 ( $84.3 \%)$ 之和为最 大 (见表4)。

表4 MNSI体格检查不同平均分值为截点时的敏感度和特异度

\begin{tabular}{ccc}
\hline 如果大于或等于则为正a & 敏感度 & 1 -特异度 \\
\hline-1 & 1.000 & 1.000 \\
1 & 0.833 & 0.157 \\
3 & 0.100 & 0.043 \\
5 & 0.022 & 0.009 \\
7 & 0.000 & 0.000
\end{tabular}

$\mathrm{a}$ : 最小界限值是最小观测检验值减 1 , 最大界限值是最大观测检验值加 1 。所有其他的界限值都是两个邻近的观 测检验值的平均值。

体格检查得分 2 分为截点时，肌电图诊断DPN结果的相关系数 kaPPa值为 0.676 （见表5）。

表5 MNSI体格检查2分截点与肌电图诊断DPN的相关性

\begin{tabular}{ccccc}
\hline 得分 & DPN & 非DPN & $\mathrm{kaPPa}$ & $P$ \\
\hline 小于2分 & 15 & 98 & & 0.000 \\
大于等于2分 & 75 & 18 & 0.676 & 0.000 \\
\hline
\end{tabular}

MNSI体格检查ROC曲线图的ROC曲线下面积为 $0.828(P<0.01)$, 见图1。

RoC Curve

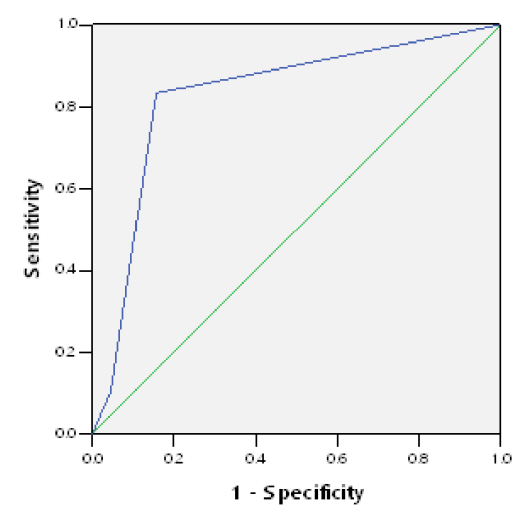

图1 MNSI体格检查ROC曲线图

$（ \mathrm{AUC}=0.828 ，$ 标准误 $=0.031 ， P=0.000 ） ， 95 \%$ CI（ $0.7670 .889 ）$

表6 MNSI问卷的得分情况

\begin{tabular}{ccc}
\hline 问卷得分 & DPN & 非DPN \\
\hline$\geqslant 0$ & $8(8.9 \%)$ & $67(57.7 \%)$ \\
$\geqslant 2$ & $34(37.7 \%)$ & $43(37.1 \%)$ \\
$\geqslant 4$ & $36(40.0 \%)$ & $6(5.2 \%)$ \\
$\geqslant 6$ & $6(6.7 \%)$ & $0(0)$ \\
$\geqslant 8$ & $6(6.7 \%)$ & $0(0)$ \\
$\geqslant 10$ & $0(0)$ & $0(0)$ \\
\hline
\end{tabular}


MNSI问卷调查不同分值时DPN和非DPN的例数见表 6, MNSI问卷调查选取1 (相当于原始数据2分) 为截断点 时，此时的灵敏度 $(91.1 \%)$ 与特异度 $(41.7 \%)$ 之和为最大 ( 见表7 )。

表7 MNSI问卷不同平均分值为截点时的敏感度和特异度

\begin{tabular}{ccc}
\hline 如果大于或等于则为正a & 敏感度 & 1-特异度 \\
\hline-1 & 1.000 & 1.000 \\
1 & 0.911 & 0.417 \\
3 & 0.533 & 0.052 \\
5 & 0.133 & 0.000 \\
7 & 0.067 & 0.000 \\
9 & 0.000 & 0.000
\end{tabular}

$\mathrm{a}$ ：最小界限值是最小观测检验值减1, 最大界限值是最大观测检验值加 1 。所有其他的界限值都是两个邻近的观 测检验值的平均值。

问卷检查得分 2 分为截点时，和肌电图相比，诊断DPN结果的相关系数kaPPa值为 0.465 （见表8）。 表8 MNSI问卷得分2分截点与肌电图诊断DPN的相关性

\begin{tabular}{ccccc}
\hline 得分 & DPN例数 & 非DPN例数 & $\mathrm{kaPPa}$ & \\
\hline 小于2分 & 8 & 67 & & \\
大于等于2分 & 82 & 49 & 0.465 & 0.000 \\
\hline
\end{tabular}

MNSI问卷调查ROC曲线图的ROC曲线下面积为 $0.838(P<0.01) ， 见$ 图2。

ROC Curve

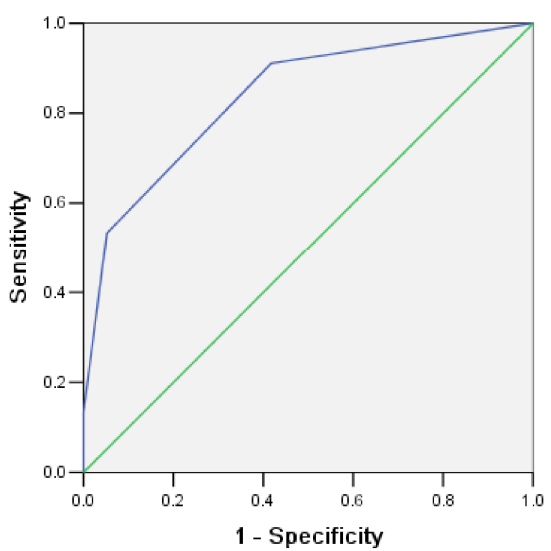

图2 MNSI问卷ROC曲线图

五、讨论

$(\mathrm{AUC}=0.838$, 标准误 $=0.028, P=0.000), 95 \% \mathrm{CI}(0.7830 .893)$

糖尿病周围神经病变导致糖尿病足的发生的重要原因之一，要引起重视。目前，我国 $80 \%$ 以上的糖尿病患者就诊 于区县级及以下基层医疗机构，基层医疗卫生机构在糖尿病足早期防治中承担重要责任，而DPN的篮查是其中重要内 容之一 ${ }^{[12]}$ 。相比三甲医院，基层卫生机构更倾向于选择类似MNSI的简单有效篮查工具。MNSI体格检查和问卷调查诊 断DPN的准确性探讨如下。

(一) MNSI体格检查的评分诊断价值

接收者工作特征曲线 (receiver operator characteristic, ROC) 采用共同的、容易解释的尺度, 通过诊断或预测的界值 对诊断系统的准确性提供了直观的视觉印象, 被认为是衡量诊断信息和诊断决策质量的最佳方法 ${ }^{[13,}{ }^{14]}$ 。一般认为, ROC 曲线下面积在 $0.5 \sim 0.7$ 之间表示诊断价值较低, 在 $0.7 \sim 0.9$ 之间表示诊断价值中等, 在 0.9 以上表示诊断价值较高 ${ }^{[13]}$ 。本研 究体格检查结果表明诊断截断点以2分时, 其在笁查DPN中具有中等诊断价值, 与张春风和Moghtaderi等的研究结果相一 致 ${ }^{[8,11]}$ 。 $\mathrm{kaPPa}$ 检验用来评价两种检验方法的一致性，一般认为 $\mathrm{K}<0.4$ 表示一致性差，K在0.4 0.75表示一致性较好， $\mathrm{K}>$ 0.75 表示一致性高 ${ }^{[15]}$ 。本研究kaPPa结果表明体格检查与神经肌电图检查诊断DPN具有较好的一致性。

（二）MNSI问卷的评分诊断价值 
图2中MNSI问卷评分ROC曲线下面积为 $0.838(P<0.01)$, 表明其在篮查DPN中具有中等诊断价值。诊断截断点 以2分较为合适, 这与张春风[7]研究结果推荐诊断截点 4 有出人, 如果本研究采取 4 分为截断点, 将会导致近一半的周围 神经病变人群被漏诊。这可能与选取的人群有关, 张春风选取的研究人群是神经内科的糖尿病患者, 本研究人群来自 内分泌科, 有关神经病变的主观症状阳性率偏低。本研究中MNSI问卷评分 $\geqslant 2$ 分的K值为 0.465 , 表明其与神经肌电图 检查诊断具有较好的一致性。

(三) MNSI量表的优越性

DPN起病隐匿, 大部分患者在早期无明显临床症状, 最终出现麻木、疼痛等感觉异常才会到医院就诊, 导致错过 最佳的诊断和治疗时机, 延误病情 ${ }^{[16]}$ 。目前, 临床上诊断DPN方法种类多, 国内仍缺乏统一标准。神经电生理（肌电 图 ) 可用于诊断周围神经病变, 但神经电生理检查价格昂贵, 操作烦琐, 难以在基层医院推广使用。密歇根神经筛查 量表和多伦多临床症状评分是国内外应用最广泛的简易篮查工具之一。孙敏研究证明多伦多临床评分系统对糖尿病周 围神经病变篮查价值较高 ${ }^{[17]}$, 但该评分系统涉及神经症状、神经反射、感觉试验进行检查, 相对比较烦琐; 而且多伦 多临床评分系统对于无症状的DPN则不具备临床检测价值 ${ }^{[18]}$ 。MNSI优越性表现为MNSI体格检查包括4项内容 (足外 观、溃疡史、踝反射和踇指振动觉）, 由于操作简单, 诊断特异性和敏感性高, 在国内外均应用广泛 ${ }^{[1]}$; MNSI两部 分内容都涉及病人足的外观情况如胼胝、指甲、感染和溃疡史等因素 ${ }^{[19]}$, 而胼胝、厚甲、溃疡史、温度觉异常和压力 觉异常等均是糖尿病足的高危因素 ${ }^{[20,21]}$, 在无症状的DPN患者中可以得到体现; MNSI操作简便, 平均耗时不超过 10 分钟, 相比多伦多临床评分平均时间 11 分钟 ${ }^{[22]}$ 有时间优势, 可以节约医护人员的宝贵时间; 密歇根神经量表评分已被 多个学者证实与客观指标糖尿病患者胫神经的超声改变亦关系密切 ${ }^{[18,23]}$ 。

六、小结

MNSI量表简单易操作, 与肌电图相比, 诊断DPN的敏感性和特异性均较好。无论是MNSI体格检查还是问卷评 分, 均可以用于糖尿病周围神经病变的篎查, 截点均为 2 分时与肌电图诊断一致性较好, 特别适合用于偏远地区或二 线城市等基层地区的糖尿病患者周围神经病变的大规模篮查。本研究未纳人门诊糖尿病患者, 结果可能会有偏移, 后 期将把门诊患者纳人展开进一步调查，使结果更具有代表性。

\section{参考文献:}

[1]中华医学会糖尿病学分会.中国2型糖尿病防治指南(2017年版)[J].中国实用内科杂志, 2018,38(04):292-344.

[2] 中华医学会神经病学分会肌电图与临床神经电生理学组, 糖尿病周围神经病诊断和治疗共识 [J]. 中华神经科杂志, 2013,46(11):787-788.

[3]潘琦,李洪梅, 邢小燕. 北京地区13家医院门诊2型糖尿病患者周围神经病变危险因素分析和篎查方法评估[J]. 北京 医学, 2018,40(11):1009-1014.

[4]Tesfaye S,Boulton AJ,Dyck PJ,etal.Diabetic neuropathies:update on definitions, diagnostic criteria, estimation of severity, and treatments. Diabetes Care, 2010,33:2285-2293.

[5]Boyraz O,Saracoglu M.The effect of obesity on the assessment of diabetic Peripheral neuropathy: A comparison of Michigan Patient version test and Michigan Physical assessment[J]. Diabetes Research and Clinical Practice, 2010, 90(3):0-260.

[6]Ferreira JP, Sartor CD, Leal Â,Sacco IC, Sato TO,Ribeiro IL,Soares AS, Cunha JE and Salvini T. The effect of Peripheral neuropathy on lower limb muscle strength in diabetic individuals[J].Clin Biomech(Bristol, Avon). 2017,43:67-73.

[7]Yi LC, Sartor CD, Souza FT and Sacco IC. Intralimb coordination Patterns in absent, mild, and severe stages of diabetic neuropathy: looking beyond kinematic analysis of gait cycle[J].PLOSONE. 2016,11(1):e0147300.

[8]Moghtaderi A,BakhshiPour A and Rashidi H . Validation of Michigan neuropathy screening instrument for diabetic Peripheral neuropathy[J]. Clin Neurol Neurosurg, 2006,108(5):477-481.

[9]Barbosa M, Saavedra A, Severo M, Maier C and Carvalho D.Validation and reliability of the Portuguese version of the Michigan Neuropathy Screening Instrument[J]. Pain Pract. 2017,17(4):514-521.

[10]Olaiya M T, Hanson R L, Kavena K G, Sinha M, Clary D, Horton M, Nelson RG and Knowler WC. Use of graded Semmes Weinstein monofilament testing for ascertaining Peripheral neuropathy in people with and without diabetes[J]. Diabetes Research and Clinical Practice. 2019,151:1-10.

[11]张春风, 谢云, 葛焕琦, 郑辉, 李宝毅, 崔壮. 密歇根神经笁查量表在糖尿病神经病变中诊断截点的观察 $[\mathrm{J}]$. 中国糖尿 病杂志, 2015,(7):602-607.

[12] 中国微循环学会糖尿病与微循环专业委员会, 中国研究型医院学会创面防治与损伤组织修复专业委员会. 糖尿病 
足基层篮查与防治专家共识[J].中国糖尿病杂志, 2019,27(6):401-407.

[13]孙振球.医学统计学[M].2版.北京: 人民卫生出版社, 2010:663-666.

[14]孙胜男,李峥.2型糖尿病风险评估工具的研究进展[J].中华护理杂志, 2009,44(9):861-864.

[15]孙振球.医学统计学 $[\mathrm{M}]$.2版.北京: 人民卫生出版社, 2010:761.

[16]陈明月,蔡慧敏, 陈江云, 张宁, 王莎. 密歇根糖尿病神经病变评分和多伦多临床评分系统在糖尿病周围神经病变中 的诊断价值研究 [J].中国全科医学, 2017,20(4):427-431.

[17]孙敏,白亚娟,方桂珍.多伦多临床评分系统对糖尿病周围神经病变笁查价值分析 [J].护理与康复, 2015,14(5):403-409.

[18]代建成, 罗平平, 梁键锋, 吴斌洪, 罗伟权, 黄志勇. 2型糖尿病下肢周围神经病变的超声形态参数与密歇根神经病变 笁查评分系统的关系研究 [J].中国医学工程, 2018,26(9):30-33.

[19]Hershey DS.Diabetic Peripheral neuropathy:evaluation and management[J].Journal for Nurse Practitioner, 2017,13(3):199204.

[20]篗良琴, 周佩如, 黄洁微, 陈玉珍, 刘雪彦, 马芳婷.广东省江门地区城乡2型糖尿病患者周围神经病变的篎查及分析 [J]. 护理学报, 2019,26(07):45-49.

[21]刘雪彦, 周佩如, 黄洁微, 唐美丽, 苏燕华, 陈庆玲.糖尿病病人下肢麻木症状与其他糖尿病足高危因素的相关性分析 [J]. 护理研究, 2019,33(13):2209-2212.

[22]杨曼,李奕平,杨荣,毛文文.多伦多临床神经病变评分在2型糖尿病周围神经病变篎查中的应用及对临床治疗的评 价[J].昆明医科大学学报, 2020,41(5):75-79.

[23]吴菲.超声评估 II 型糖尿病患者胫神经改变与密歇根䈨查量表评分的关系[J]. 实用医院临床杂志, 2018,15(4):205-207. 